\title{
Implementation of CRC Algorithm in UHF RFID Test System Based on Labview
}

\author{
Junbao Zhang $^{\mathrm{a}}$ and Hai Gao ${ }^{\mathrm{b}}$
}

School of Automation and Electrical Engineering, University of Science and Technology Beijing, Beijing 100083, China

as20160636@xs.ustb.edu.cn, bgaohai@ustb.edu.cn

Keywords: UHF RFID, CRC-5, CRC-16, Labview.

\begin{abstract}
This paper introduces the principle of Cyclic Redundancy Check algorithm in Ultra High Frequency Radio Frequency Identification conformance test system. According to the CRC-5 and CRC-16 hardware schematics introduced in the EPC Global Class 1 Gen2 and ISO/IEC 18000-6C protocol standards, the CRC algorithm is designed and implemented by Labview in a new way, different from traditional methods. Finally, we verify the correctness of the algorithm by a Query command and a Select command.
\end{abstract}

\section{Introduction}

Because of a variety of reasons, data received may be wrong in the process of transmission. Therefore, we need to check the correctness of the data when we receive it. Cyclic Redundancy Check (CRC) has become a widely used error checking method in communication system due to its advantages of powerful error correction and easy implementation. In the Ultra High Frequency Radio Frequency Identification (UHF RFID) conformance test system, the reader and the tag communicate with each other and then are performed conformance testing according to the standard. CRC is one of the most critical and important parts of UHF RFID conformance testing systems. If there is no CRC or the data verification result is wrong, the system will not perform the next communication and consistency analysis. In the communication process, the CRC algorithm is need to determine the correctness of data transmission. For example, Select, Req_RN, Read, Write, Kill, Lock, Access and other commands use the CRC-16 to check and the Query command uses the CRC-5 to check [1].

\section{Principle of the CRC algorithm}

The ISO/IEC 18000-6C protocol [2] and EPC Global Class1 Gen2 protocol [3] are two main internationally recognized standards for UHF RFID conformance testing, both of which have fused well in UHF test protocols. In addition, the principle of CRC algorithm is put forward clearly in the both of protocols. The basic principle is similar to those in other application fields, but the CRC algorithm of UHF RFID conformance test system is different from other application fields in terms of design and implementation.

Let $\mathrm{m}(\mathrm{x})$ be the polynomial of the data information for transmission, $\mathrm{g}(\mathrm{x})$ is the contract polynomial of the highest power $r$, and move $m(x)$ to the left by $r$ to get $M(x)$ :

$\mathrm{M}(\mathrm{x})=\mathrm{m}(\mathrm{x}) \cdot 2^{\mathrm{r}}$

The shifted information polynomial and the contract polynomial perform modulo-division (exclusive OR operation), then we get Quotient $\mathrm{Q}(\mathrm{x})$ and remainder $\mathrm{r}(\mathrm{x})$ :

$\frac{\mathrm{M}(\mathrm{x})}{\mathrm{g}(\mathrm{x})}=\frac{\mathrm{Q}(\mathrm{x}) \cdot \mathrm{g}(\mathrm{x})+\mathrm{r}(\mathrm{x})}{\mathrm{g}(\mathrm{x})}=\mathrm{Q}(\mathrm{x})+\frac{\mathrm{r}(\mathrm{x})}{\mathrm{g}(\mathrm{x})}$

The data information and the verification information are composed of the transmission data $\mathrm{T}(\mathrm{x})$, and the new data is sent out:

$\mathrm{T}(\mathrm{x})=\mathrm{M}(\mathrm{x})+\mathrm{r}(\mathrm{x})$

The Receiver receives the data $\mathrm{R}(\mathrm{x})$, if the data is correct in the transmission process, then:

$\mathrm{R}(\mathrm{x})=\mathrm{T}(\mathrm{x})=\mathrm{M}(\mathrm{x})+\mathrm{r}(\mathrm{x})=\mathrm{Q}(\mathrm{x}) \cdot \mathrm{g}(\mathrm{x})+\mathrm{r}(\mathrm{x})+\mathrm{r}(\mathrm{x})$

The received data and the contract polynomial perform modulo-division again, and we obtained: 
$\frac{\mathrm{R}(\mathrm{x})}{\mathrm{g}(\mathrm{x})}=\frac{\mathrm{Q}(\mathrm{x}) \cdot \mathrm{g}(\mathrm{x})+\mathrm{r}(\mathrm{x})+\mathrm{r}(\mathrm{x})}{\mathrm{g}(\mathrm{x})}=\mathrm{Q}(\mathrm{x})$

From the formula (5) can be seen, If the remainder is 0 after checking, the data is transferred correctly. On the contrary, the data transmission is wrong.

\section{Design and Implementation}

\subsection{CRC-5}

In the UHF RFID conformance test system, Query command requires to be checked by CRC-5. Query command as shown in Table 1, CRC-5 checks 17-bit binary data from the Command to the end of Q, which is defined in Table 2.

\begin{tabular}{|c|c|c|}
\hline & Bit & Descrintion \\
\hline Command & 4 & 1000 \\
\hline DR & 1 & $0: \mathrm{DR}=8 ; 1: \mathrm{DR}=64 / 3$ \\
\hline M & 2 & $\begin{array}{l}00: M=1 ; 01: M=2 \\
10: M=4 ; 11: M=8\end{array}$ \\
\hline TRext & 1 & $\begin{array}{l}0: \text { No pilot tone } \\
1: \text { pilot tone }\end{array}$ \\
\hline Sel & 2 & $\begin{array}{l}\text { 00:All;01:All } \\
\text { 10: Sl;11:SL }\end{array}$ \\
\hline Session & 2 & $\begin{array}{l}00: S 0 ; 01: S 1 \\
10: S 2 ; 11: S 3\end{array}$ \\
\hline Target & 1 & $0: A ; 1: B$ \\
\hline $\mathrm{Q}$ & 4 & $0-15$ \\
\hline CRC-5 & 5 & - \\
\hline
\end{tabular}

Table 2 Definition of CRC-5

\begin{tabular}{|c|c|c|c|}
\hline Polynomial & Length & Preset Value & Remainder \\
\hline $\mathrm{X}^{5}+\mathrm{X}^{3}+1$ & 5 & 010012 & $00000_{2}$ \\
\hline
\end{tabular}

The hardware principle of CRC-5[3]is shown in Fig.1, and the preset value 010012 is loaded into the CRC registers (C [4:0]), and then the Query command data bits are serially input from the Data side in MSB priority mode until the last One, C[4:0] save CRC-5 code of the data for Query command.

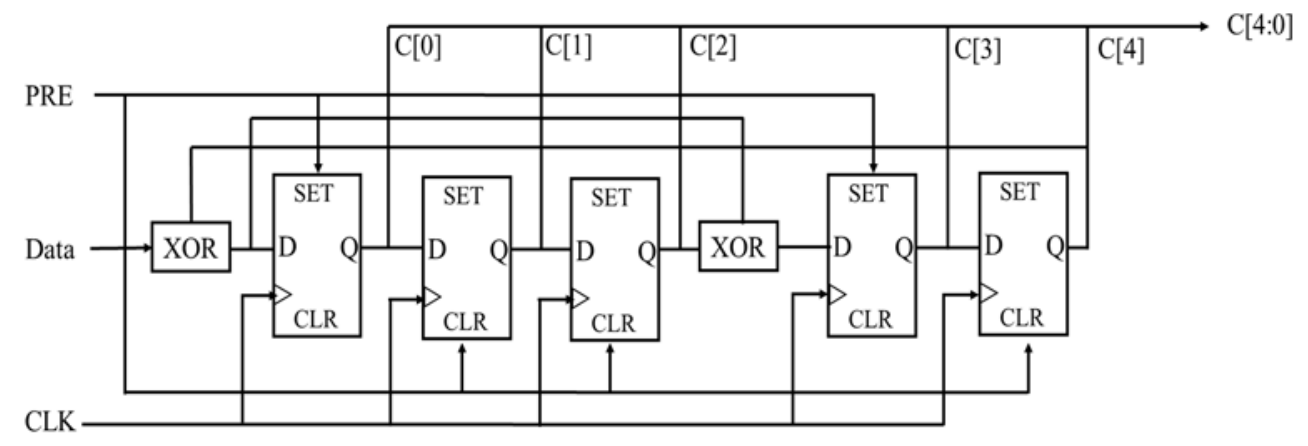

Fig. 1 The hardware principle of CRC-5

According to the schematic diagram, Human Machine Interface (HMI) is designed in the front panel of the Labview as shown in Fig.2, and the program panel as shown in Fig.3. 
CRC-5 in UHF RFID Test

Polynomial: $\mathrm{x}^{\mathbf{5}}+\mathrm{x}^{\mathbf{3}}+\mathbf{1}$

preset value: $\mathbf{0 1 0 0 1}_{2}$

Remainder: $\mathbf{0 0 0 0 0}_{2}$

Data

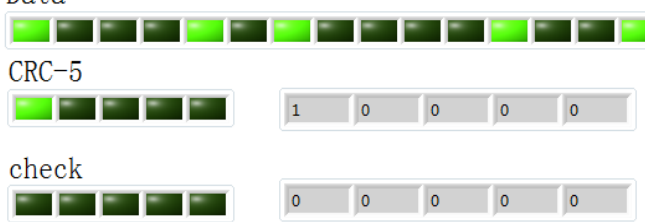

Fig.2 HMI of CRC-5

We select a commonly used Query command: 100010100001001002 . Using recursive call ideas to use Data+CRC-5 as new validation data, then we get the CRC-5 is 000002 , which proves the correctness of the algorithm.

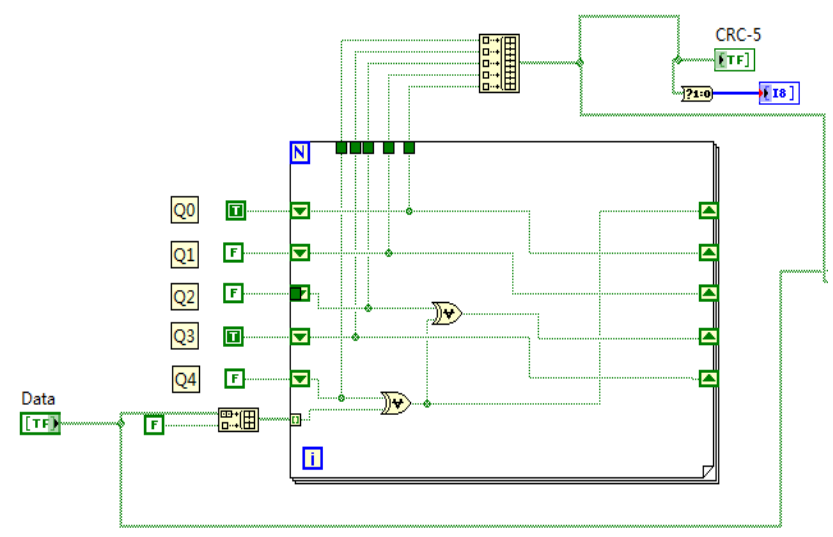

Fig.3 program panel of CRC-5

\subsection{CRC-16}

In the process of radio frequency identification, most of the commands need to be checked by CRC-16. The select command (Table 3) is the most important and commonly used command. CRC16 checks variable binary data from the Command to the end of Truncate which is defined in Table 4. CRC-16 has a variety of check polynomials, such as $X^{16}+X^{15}+X^{2}+1$ (8005), $X^{16}+X^{12}+X^{5}+1$ (CCITT). UHF RFID test protocol specifies the CRC-16 parameters as shown in Table 4.

Table 3 Select Command

\begin{tabular}{|c|c|c|}
\hline & Bit & Description \\
\hline Command & 4 & 1010 \\
\hline Target & 3 & $\begin{array}{c}\text { 000:S0;001:S1;010:S2 } \\
\text { 011:S3;100:SL;101:RFU } \\
\text { 110:RFU;111:RFU }\end{array}$ \\
\hline Action & 3 & $\begin{array}{c}\text { 000:Sure SL;001:Sure SL } \\
\text { 010:Nothing;011: negative SL }\end{array}$ \\
\hline MenBank & 2 & $\begin{array}{l}\text { 00:RFU;01EPC } \\
\text { 10:TID;11:User } \\
\end{array}$ \\
\hline Pointer & EBV & Start mask address \\
\hline Length & 8 & Length of mask \\
\hline Mask & variable & Value of mask \\
\hline Truncate & 1 & 0: Forbidden;1:Open \\
\hline CRC-16 & 16 & - \\
\hline
\end{tabular}


Table 4 Definition of CRC-16

\begin{tabular}{|c|c|c|c|}
\hline Polynomial & Length & Preset Value & Remainder \\
\hline $\mathrm{X}^{16}+\mathrm{X}^{12}+\mathrm{X}^{5}+1$ & 16 & FFFF $_{\mathrm{h}}$ & $1 \mathrm{D} 0 \mathrm{~F}_{\mathrm{h}}$ \\
\hline
\end{tabular}

The hardware principle of the CRC-16 [3] is shown in Fig.4. The preset value $\mathrm{FFFF}_{\mathrm{h}}$ is loaded into the CRC registers $(\mathrm{C}[15: 0])$, and then the Select command data bits are serially input from the Data side in MSB priority mode until the last One, C[15:0] save check anti-code of the data for the Select command. we get CRC-16 code by reversing.

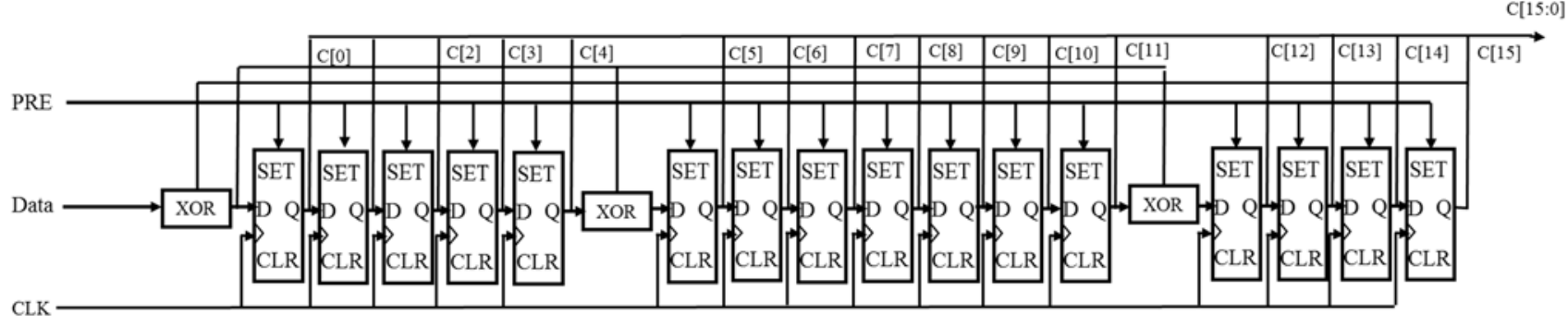

Fig.4 The hardware principle of CRC-16

According to the schematic diagram, HMI in the front panel of the labview as shown in Fig.5, and the program panel in Fig.6.

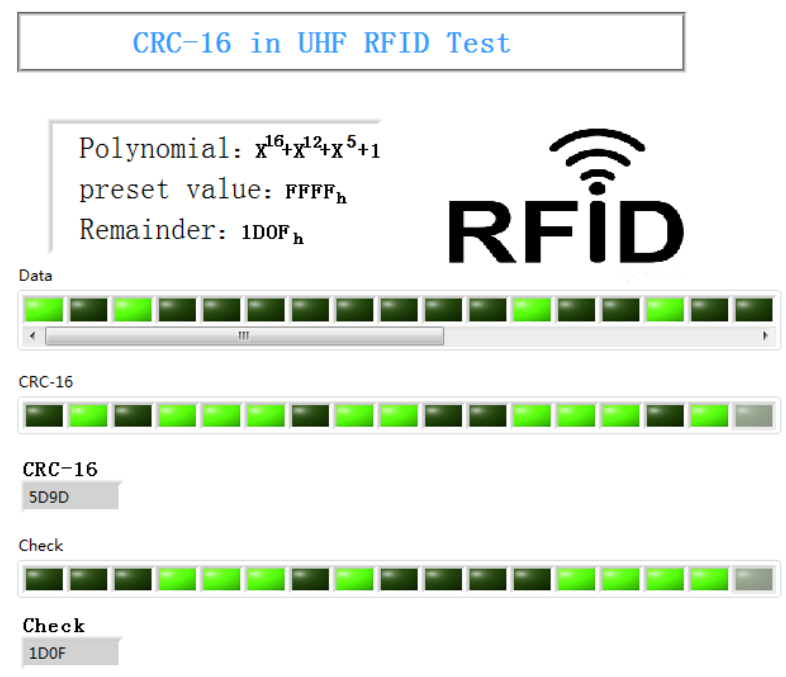

Fig.5 HMI of CRC-16

We choose a commonly used Select command: 101000000001001000000000000002 , then we get CRC-16 code is $5 \mathrm{D} 9 \mathrm{D}$. According to the idea of recursive call,we use Data+CRC-16 as new check data from the data side, then stored content in register $C[15: 0]$ is no longer negated, and the received $\mathrm{CRC}-16$ is $1 \mathrm{D} 0 \mathrm{~F}_{\mathrm{h}}$. The result proves the design is correct.

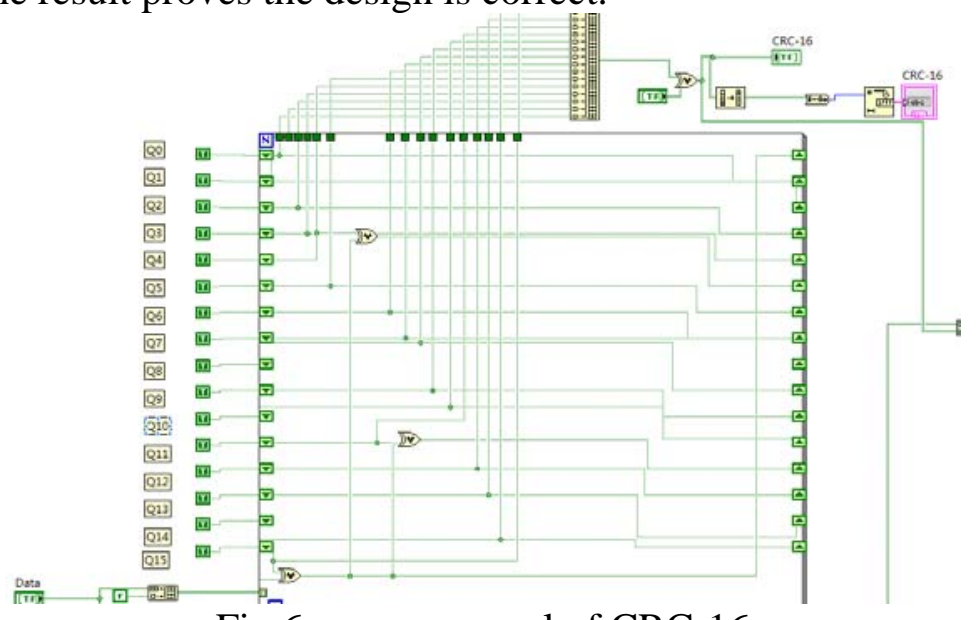

Fig.6 program panel of CRC-16 


\section{Conclusion}

This paper introduces a new design of CRC algorithm in UHF RFID Conformance testing system. The implement of CRC algorithm is based on the ISO/IEC 18000-6C and EPC Global Class 1Gen 2 test protocols. Using the labview software platform, an effective design different from traditional methods is completed, and finally the correctness is verified.

\section{References}

[1] Bin Bin Liao, Zhi Jin Zhao, Fu Hong Zhang, Implementation of CRC Algorithm in EPCglobal C1 Gen2 Standard, J. Computer Engineering, 2008, 24: 046.

[2] ISO/IEC 18000-6 . Information Technology-Radio frequency Identification for Item Management-Part 6: Parameters for Air Interface Communications at $860 \mathrm{MHz}$ to $960 \mathrm{MHz}$ (2006).

[3] EPC $^{\mathrm{TM}}$ Radio-Frequency Identity Protocols Class-1 Generation-2 UHF RFID Protocol for Communications at $860 \mathrm{MHz}-960 \mathrm{MHz}(2004)$. 